\title{
Expression of NGAL and NGALR in human embryonic, fetal and normal adult tissues
}

\author{
PI-XIAN ZHANG ${ }^{1 *}$, FA-REN ZHANG $^{2 *}$, JIAN-JUN XIE $^{1}$, LI-HUA TAO $^{3}$, ZHUO LÜ $^{1}$, \\ XIU-E XU ${ }^{2}$, JIAN SHEN ${ }^{3}$, LI-YAN XU ${ }^{2}$ and EN-MIN LI ${ }^{1}$ \\ ${ }^{1}$ Department of Biochemistry and Molecular Biology; ${ }^{2}$ Institute of Oncologic Pathology, \\ The Key Immunopathology Laboratory of Guangdong Province; ${ }^{3}$ Department of Pathology, \\ Medical College of Shantou University, Shantou, Guangdong 515041, P.R. China
}

Received February 10, 2012; Accepted June 12, 2012

DOI: $10.3892 / \mathrm{mmr} .2012 .980$

\begin{abstract}
This study investigated the distribution of neutrophil gelatinase-associated lipocalin (NGAL) and neutrophil gelatinase-associated lipocalin receptor (NGALR) in human embryonic, fetal and normal adult tissues. Tissue microarray technology was used to perform immunohistochemical examination on human embryos, fetuses at 4-22 weeks of gestation and adult tissue specimens. Results demonstrated that during the development of the nervous system, NGALR was prevalent in the neural tube and cerebrum, and NGAL was only detected in the stellate cells of the cerebrum and the Purkinje cells of the cerebellum. NGAL and NGALR were expressed in the lung alveolar epithelium and in the gastrointestinal tract in embryos, but were almost undetectable in later developmental stages. In the embryonic adrenal glands, the two proteins demonstrated moderate positivity in the cortex and the medulla. In adults, NGAL was particularly present in the cells of the inner and outer layers of the cortex and was absent in the medulla, while NGALR exhibited strong positivity in the cortex and the medulla. Evident expression of NGAL and NGALR was observed throughout development in the neutrophil-rich sites, the renal tubule epithelium and certain gland epithelia of the gastrointestinal tract, but was undetectable in the heart, liver and thyroid gland. Taken together, these results demonstrated that the expression of NGAL and NGALR was time-specific and highly tissue-specific. Correlations between their expression in embryogenesis and carcinogenesis should be examined.
\end{abstract}

Correspondence to: Professor En-Min Li or Professor Li-Yan Xu, Department of Biochemistry and Molecular Biology, Medical College of Shantou University, No. 22 Xinling Road, Shantou, Guangdong 515041, P.R. China

E-mail:nmli@stu.edu.cn

E-mail: liyanxu1130@yahoo.com.cn

${ }^{*}$ Contributed equally

Key words: neutrophil gelatinase-associated lipocalin, neutrophil gelatinase-associated lipocalin receptor, embryo, development, tissue microarray, immunohistochemistry

\section{Introduction}

Neutrophil gelatinase-associated lipocalin (NGAL), a member of the lipocalin family of small secreted proteins, was originally identified as a protein stored in specific granules of human neutrophils (1). Besides being expressed in neutrophils, NGAL is expressed in the majority of tissues normally induced in epithelial cells during inflammation $(2,3)$, delivering iron to cells during formation of the tubular epithelial cells of the primordial kidney (4), and protecting against acute ischemic renal injury, thus it is involved in apoptosis as a survival factor $(5,6)$. NGAL has been identified in a variety of normal and pathological human tissues. A cell type-specific pattern of expression has been observed in the bronchus, stomach, small intestine, pancreas, kidney, prostate gland and thymus (3). Expression of NGAL has also been detected in human cancers, including colorectal (2), breast (7), pancreatic (8), bladder (9), ovarian (10), esophageal squamous cell carcinoma (11) and gastric carcinoma (12), and its homologue is also expressed during chicken embryo development in hypertrophic cartilage (13), and in the hypertrophic region of growth plate cartilage in developing rat embryos (14).

The receptor of the homologue of NGAL in mice 24 p3 (24 p3R) was identified and the corresponding human receptor (NGALR) was also found $(15,16)$. NGALR was reported to have a function dependent on the status of NGAL. NGAL and NGALR constitute a novel iron delivery pathway that is crucial to the survival, growth and maturation of cells (17). This observation led investigators to explore the co-expression of NGAL and NGALR. NGALR expression in esophageal squamous cell carcinoma was found to be significantly higher compared to normal esophageal epithelium (18). More recently, we found that NGAL and NGALR might be involved in the progression of esophageal squamous cell carcinoma and gliomas, and their expression can be considered as independent prognostic factors in these tumors $(19,20)$. However, little is known regarding the systematic demonstration of NGAL and NGALR expression in various human organs at different developmental stages.

To improve the understanding of the function of these two proteins in human development, we aimed to demonstrate the expression of NGAL and NGALR in human embryo, fetus 
and normal adult tissues by employing tissue microarray technology and immunohistochemical staining.

\section{Materials and methods}

Samples. Forty human samples consisting of 3 embryos, 11 fetuses and 26 postnatal specimens were examined in the study (Table I). Intact embryos and fetuses were obtained from the Department of Gynaecology and Obstetrics, Central Hospital of Shantou, Shantou, China. Samples were collected from 14 healthy pregnant females undergoing elective termination of pregnancy at 4-22 weeks of gestation. Specimens were fixed immediately in $4 \%$ buffered-formalin solution, and visible organs were embedded in paraffin blocks. Normal human tissue sections (from autopsy specimens) were obtianed from the Department of Forensic Medicine, Shantou University Medical College, Shantou, China. The specimens were collected between 2002 and 2005. Tissues from the cerebellum, cerebrum, thymus, lung, trachea, heart, esophagus, stomach, large intestine, small intestine, liver, pancreas, kidney, spleen, thymus, lymph-node, pituitary, adrenal gland and thyroid gland were collected. The study was conducted with permission from the local ethics committees.

Construction of tissue microarrays. Representative regions from each tissue were selected from hematoxylin- and eosin-stained sections and marked on individual paraffin blocks. Samples were selected from the specimens with a large quantity of tissue available for correlative studies. Two tissue cores, measuring $1.8 \mathrm{~mm}$ in diameter and ranging in length from 1.0 to $3.0 \mathrm{~mm}$ depending on the depth of the tissue in the donor block, were obtained from each specimen. Each core was precisely arrayed into a new paraffin block. The microarrays were serially sectioned $(4 \mu \mathrm{m})$, and stained with hematoxylin and eosin to verify tissue sampling and completeness. Unstained sections were baked overnight at $56^{\circ} \mathrm{C}$ prior to immunohistochemistry.

Immunohistochemical staining. Slides were dried in an oven $\left(55-60^{\circ} \mathrm{C}\right)$ and the paraffin was removed through several changes of xylene. The slides were hydrated through a series of graded alcohol to water followed by incubation with $3 \%$ hydrogen peroxide for $10 \mathrm{~min}$. For antigen retrieval, the slides were autoclaved in $0.01 \mathrm{M}$ citrate buffer $\left(\mathrm{pH} \mathrm{6.0)}\right.$ ) at $120^{\circ} \mathrm{C}$ for 3 min. Sections were then incubated with $10 \%$ normal goat serum in phosphate-buffered saline (PBS) for $15 \mathrm{~min}$ at room temperature to block non-specific binding. After rinsing with PBS, the slides were incubated at $4^{\circ} \mathrm{C}$ overnight with mouse anti-human NGAL monoclonal antibody (1:50 dilution; R\&D Systems, Minneapolis, MN, USA) or rabbit anti-human NGALR polyclonal antibody (1:10 dilution, Beijing Biosynthesis Biotechnology Co., Ltd., Beijing, China). After further rinsing with PBS, the tissue sections were incubated for $15 \mathrm{~min}$ at room temperature with polymer helper solution (Polymer Detection System kit, Golden Bridge International, Inc., Mukilteo, WA, USA), and then rinsed with PBS. The slides were then incubated for $20 \mathrm{~min}$ at room temperature with streptavidin peroxidase-conjugated goat anti-mouse IgG (Polymer Detection System kit). Subsequently, the slides were
Table I. Description of specimens.

Human stage

Number of cases

\begin{tabular}{lr}
\hline Embryo & 3 \\
4 weeks' gestation & 1 \\
$5-8$ weeks' gestation & 2 \\
Fetus & 11 \\
9-12 weeks' gestation & 5 \\
13-16 weeks' gestation & 3 \\
17-22 weeks' gestation & 3 \\
Postnatal stages & 26 \\
Neonate (<28 days) & 5 \\
1-19 years & 6 \\
20-39 years & 11 \\
$>40$ years & 4 \\
\hline
\end{tabular}

Table from ref. 23 .

stained with $0.003 \%$ 3,3-diaminobenzide tetrahydrochloride and $0.005 \%$ hydrogen peroxide in $0.05 \mathrm{M}$ Tris- $\mathrm{HCl}(\mathrm{pH} 7.2)$, and counterstained using Mayer's hematoxylin prior to being dehydrated and mounted. A metastatic esophageal carcinoma previously demonstrated to have immunoreactivity was used as a positive control in each series of experiments (11). Negative controls were prepared by substituting PBS for primary antibody.

NGAL-positive samples were defined as those demonstrating brown signals in the cell membrane and cytosol. Immunoreactivity was measured semiquantitatively using a scale from (-) to $(+++)$, where $(-)$ represented no detectable immunostaining; $(+)$ that $<25 \%$ of the cells were reactive; $(++)$ that $>50 \%$ of the cells were reactive; and $(+++)$ that $>50 \%$ of the cells were reactive. Finally, scales of $(-),(+),(++)$ and $(+++)$ were defined as negative, weakly positive, moderately positive and strongly positive staining, respectively.

\section{Results}

The distribution of NGAL and NGALR in normal human tissues as determined by immunostaining. Expression of NGAL and NGALR was homogeneous in the postnatal stages; however, differential expression was observed during embryonic and fetal development (Table II).

Nervous system. In the development of the nervous system, the expression of NGAL was undetectable in the early embryos, but detectable in the later developmental stages, and NGALR protein was detected throughout human development. In the early embryos, the neuroepithelium of the neural tube demonstrated NGAL-negative (Fig. 1A) and NGALR-positive expression (Fig. 1B). In the fetuses and adults, NGAL and NGALR immunoreactivities were detected in stellate cells of the cerebrum, but not in glial cells (Fig. 1C and D). In the cerebellum, no immunoreactivity was found in any cells of the Purkinje cell, granular or molecular layer at 8-12 weeks' gestation (Fig. 1E and F); however, the Purkinje cells demonstrated 
Table II. Distribution of NGAL and NGALR in normal human tissues determined by immunostaining.

\begin{tabular}{|c|c|c|c|c|c|c|c|c|}
\hline \multirow[b]{3}{*}{ Organ/system } & \multirow[b]{3}{*}{ Tissue } & \multicolumn{7}{|c|}{ NGAL/NGALR } \\
\hline & & \multirow{2}{*}{$\begin{array}{c}\text { Embryo } \\
\begin{array}{c}4-8 \\
\text { weeks' }\end{array} \\
\text { gestation }\end{array}$} & \multicolumn{2}{|c|}{ Fetus } & \multicolumn{4}{|c|}{ Postnatal stages } \\
\hline & & & $\begin{array}{c}\text { 8-16 } \\
\text { weeks' } \\
\text { gestation }\end{array}$ & $\begin{array}{c}\text { 17-22 } \\
\text { weeks' } \\
\text { gestation }\end{array}$ & $\begin{array}{l}\text { Neonate } \\
(<28 \text { days })\end{array}$ & $\begin{array}{c}1-19 \\
\text { years }\end{array}$ & $\begin{array}{l}20-39 \\
\text { years }\end{array}$ & $\begin{array}{c}>40 \\
\text { years }\end{array}$ \\
\hline \multicolumn{9}{|l|}{ Nervous system } \\
\hline Nerve cell & & $-/+$ & $-/+++$ & $+/+++$ & $+/+++$ & $+/+++$ & $+/+++$ & $+/+++$ \\
\hline Nerve fiber & & $-/+$ & $-/++$ & $-/++$ & $-/++$ & $-/++$ & $-/++$ & $-/++$ \\
\hline \multicolumn{9}{|l|}{ Cardiovascular system } \\
\hline Heart & & NA & $-/-$ & $-/-$ & $-/-$ & $-/-$ & $-/-$ & $-/-$ \\
\hline Artery & & NA & $-/-$ & $-/-$ & $-/-$ & $-/-$ & $-/-$ & $-/-$ \\
\hline \multicolumn{9}{|l|}{ Respiratory system } \\
\hline Lung & & NA & $++/++$ & $++/++$ & $-/++$ & $-/++$ & $-/++$ & $-/++$ \\
\hline Trachea & & NA & $++/++$ & $++/++$ & $-/-$ & $-/-$ & $-/-$ & $-/-$ \\
\hline \multicolumn{9}{|l|}{ Alimentary system } \\
\hline Gastrointestinal tract & & $++/++$ & $++/++$ & $-/-$ & $-/-$ & $-/-$ & $-/-$ & $-/-$ \\
\hline Liver & & $-/-$ & $-/-$ & $-/-$ & $-/-$ & $-/-$ & $-/-$ & $-/-$ \\
\hline Pancreas & & NA & $-/+++$ & $-/+++$ & $-/+++$ & $-/+++$ & $-/+++$ & $-/+++$ \\
\hline \multicolumn{9}{|l|}{ Genitourinary system } \\
\hline \multicolumn{9}{|l|}{ Kidney } \\
\hline & Glomerulus & NA & $+/+$ & $+/+$ & $+/+$ & $+/+$ & $+/+$ & $+/+$ \\
\hline & Proximal tubule & NA & $++/+++$ & $++/+++$ & $++/+++$ & $++/+++$ & $++/+++$ & $++/+++$ \\
\hline & Distal tubule & NA & $+++/++$ & $+++/++$ & $+++/++$ & $+++/++$ & $+++/++$ & $+++/++$ \\
\hline & Collecting duct & NA & $+++/++$ & $+++/++$ & $+++/++$ & $+++/++$ & $+++/++$ & $+++/++$ \\
\hline Urothelium & & NA & NA & NA & $-/-$ & $-/-$ & $-/-$ & $-/-$ \\
\hline Prostate gland & & NA & NA & NA & $-/-$ & $-/-$ & $-/-$ & $-/-$ \\
\hline \multicolumn{9}{|l|}{ Endocrine system } \\
\hline \multicolumn{9}{|l|}{ Adrenal gland } \\
\hline & Zona glomerulosa & NA & $++/++$ & $++/++$ & $+++/+++$ & $+++/+++$ & $+++/+++$ & $+++/+++$ \\
\hline & Zona fasciculata & NA & $++/++$ & $++/++$ & +/+++ & +/+++ & +/+++ & +/+++ \\
\hline & Zona reticularis & NA & $++/++$ & $++/++$ & $+++/+++$ & $+++/+++$ & $+++/+++$ & $+++/+++$ \\
\hline & Medulla & NA & $++/++$ & $++/++$ & $-/+++$ & -/+++ & -/+++ & -/+++ \\
\hline Thyroid gland & & NA & NA & NA & $-/-$ & $-/-$ & $-/-$ & $-/-$ \\
\hline Pituitary & & NA & NA & NA & $++/++$ & $++/++$ & $++/++$ & $++/++$ \\
\hline \multicolumn{9}{|l|}{ Immune system } \\
\hline Spleen & & NA & $++/++$ & $++/++$ & $++/++$ & $++/++$ & $++/++$ & $++/++$ \\
\hline Lymphoid node & & NA & NA & NA & $+/+$ & $+/+$ & $+/+$ & $+/+$ \\
\hline Thymus & & NA & NA & $++/++$ & $++/++$ & NA & NA & NA \\
\hline \multicolumn{9}{|l|}{ Skin } \\
\hline & Basal layer & NA & $++/++$ & $++/++$ & $++/++$ & $++/++$ & $++/++$ & $++/++$ \\
\hline
\end{tabular}

Immunoreactivity was measured semi-quantitatively using a scale from (-) to $(+++)$. (-), no immunostaining; $(+),<25 \%$ of the cells were reactive; $(++), 25-50 \%$ of the cells were reactive; $(+++),>50 \%$ of the cells were reactive. Scales of $(-),(+),(++)$ and $(+++)$ were defined as negative, weakly positive, moderately positive and strongly positive staining, respectively. NGAL, neutrophil gelatinase-associated lipocalin; NGALR, neutrophil gelatinase-associated lipocalin receptor; N/A, no analysis

pronounced immunoreactivity in the later developmental stages (Fig. 1G and $\mathrm{H}$ ).

Alimentary system. The expression pattern of NGAL and NGALR was similar to the gastrointestinal development. At 6-8 weeks' gestation, the two proteins were expressed in cells of the gastrointestinal tract (Fig. 2A and B). In later developmental stages, the proteins were observed in certain cells of the glandular epithelium, including the Paneth's and gastric parietal cells (Fig. 2C and D). In the liver, NGAL and NGALR were weakly positive. In the pancreas, the acini and tubular epithelium of the glandular pancreatic tissue 

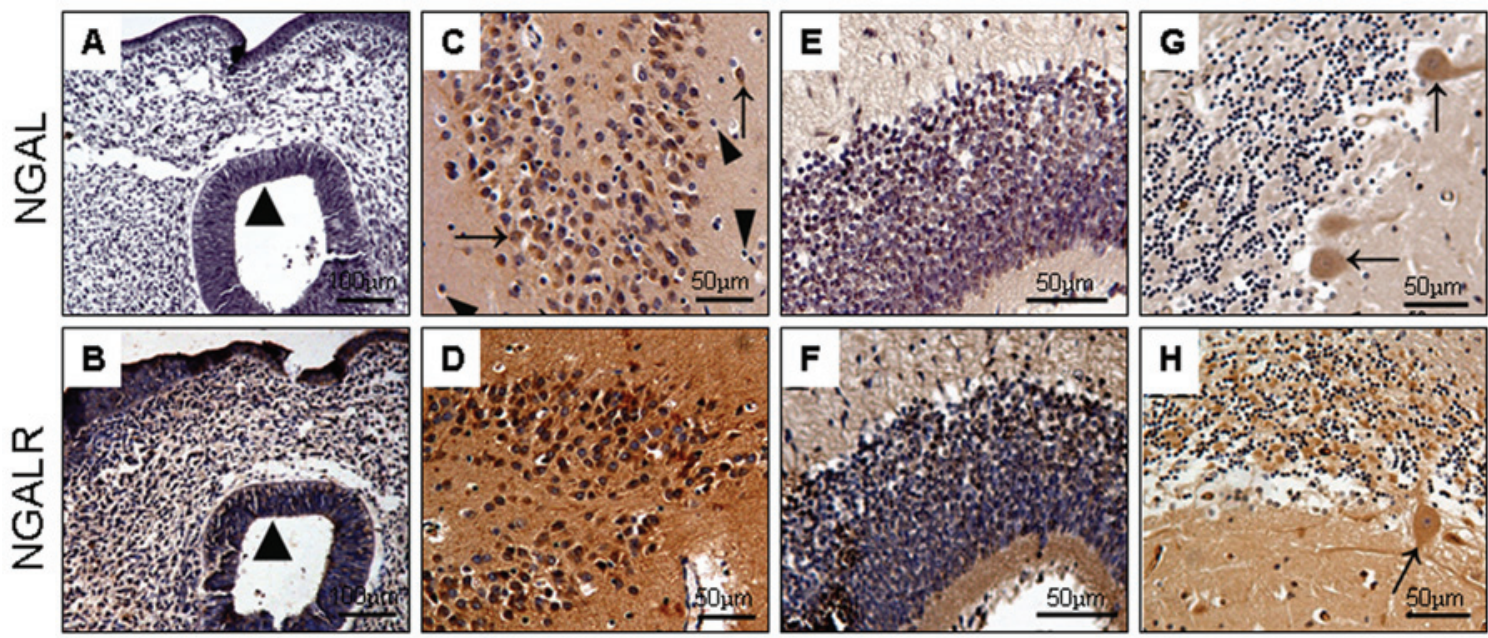

Figure 1. Expression of NGAL and NGALR in the nervous system. (A and B) At 4 weeks' gestation, the neuroepithelium (triangle) of the neural tube demonstrated NGAL-negative and NGALR-positive expression. (C and D) In the adult cerebrum, NGAL and NGALR were detected in the stellate cells (arrows), but not in glial cells (arrowheads). (E and F) In the cerebellum, no reactivity was found in any cells of the Purkinje cell layer, granular layer or molecular layer at 12 weeks' gestation. ( $\mathrm{G}$ and $\mathrm{H}$ ) The Purkinje cells (arrows) of the cerebellum demonstrated pronounced immunoreactivity in the later developmental stages. NGAL, neutrophil gelatinase-associated lipocalin; NGALR, neutrophil gelatinase-associated lipocalin receptor.
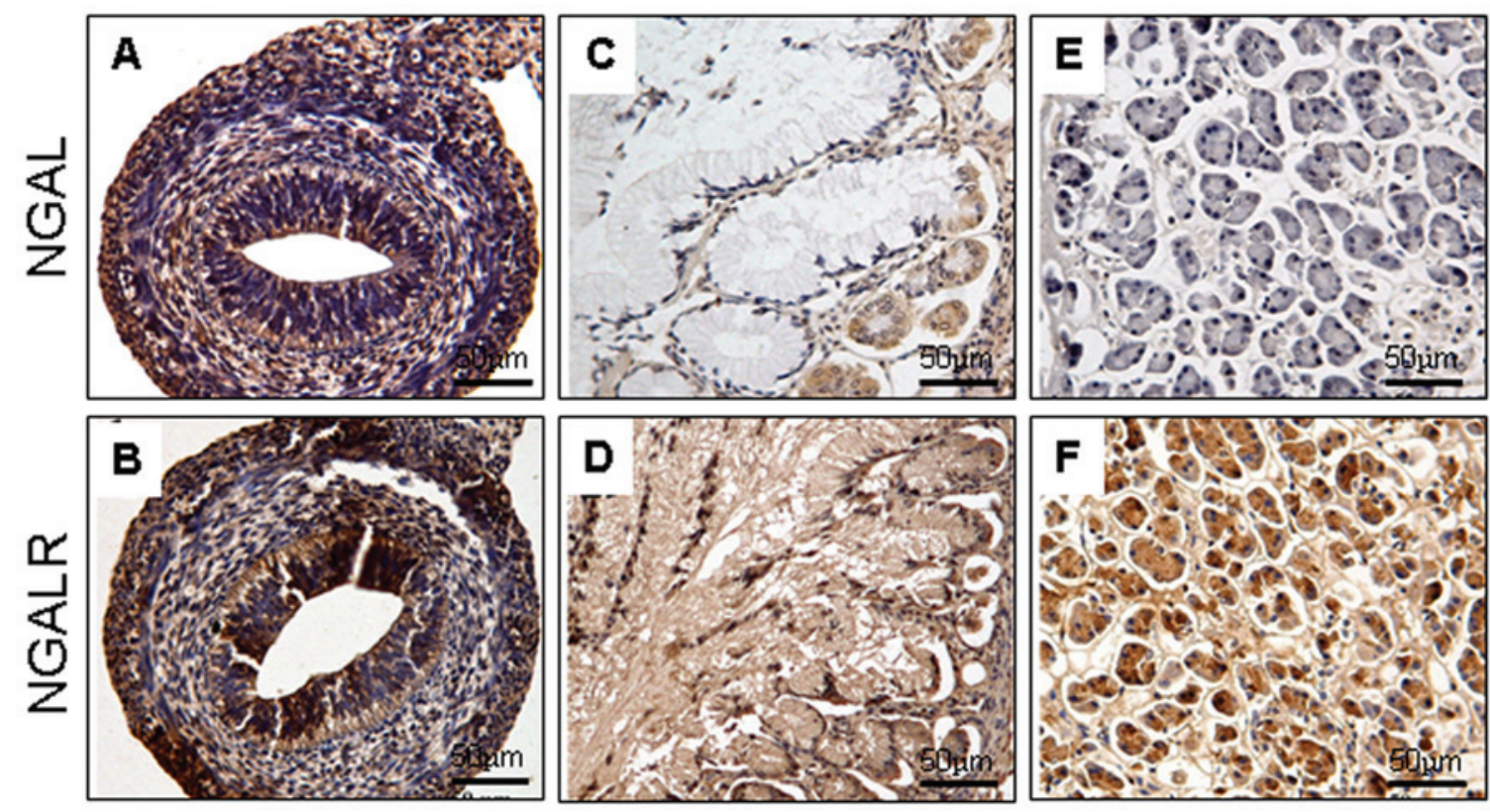

Figure 2. NGAL and NGALR expression in the alimentary system. (A and B) At 8 weeks' gestation, NGAL and NGALR were expressed in cells of the gastrointestinal tract. (C and D) In later developmental stages, the proteins were observed in certain cells of the glandular epithelium, including Paneth's cells. (E and F) In the pancreas, the acini demonstrated NGAL-negative and NGALR-positive expression. NGAL, neutrophil gelatinase-associated lipocalin; NGALR, neutrophil gelatinase-associated lipocalin receptor.

exhibited NGAL-negative (Fig. 2E) and NGALR-positive expression (Fig. 2F).

Endocrine system. During the development of the endocrine system, the expression of NGAL and NGALR was detectable in certain cells of the anterior pituitary, but undetectable in the follicular and C-cells of the thyroid gland (Fig. 3A and B). In the adrenal gland, the two proteins demonstrated a moderately positive reaction at 8-22 weeks' gestation in the cortex and the medulla (Fig. 3C and D). However, in later stages of development, NGAL was particularly demonstrated in cells of the inner and outer layers of the cortex, decreased in the zona fasciculata and entirely absent in the medulla (Fig. 3E), while NGALR demonstrated strong positivity in the cortex and the medulla (Fig. 3F).

Expression of NGAL and NGALR in other systems. In the cardiovascular system, NGAL and NGALR produced no or weak reactivity in the vascular endothelial and myocardial cells (Fig. 4A and B). In the respiratory system, NGAL and NGALR were most prevalent in the epithelium of the alveolar gland in the early embryos (Fig. 4C and D), but not 

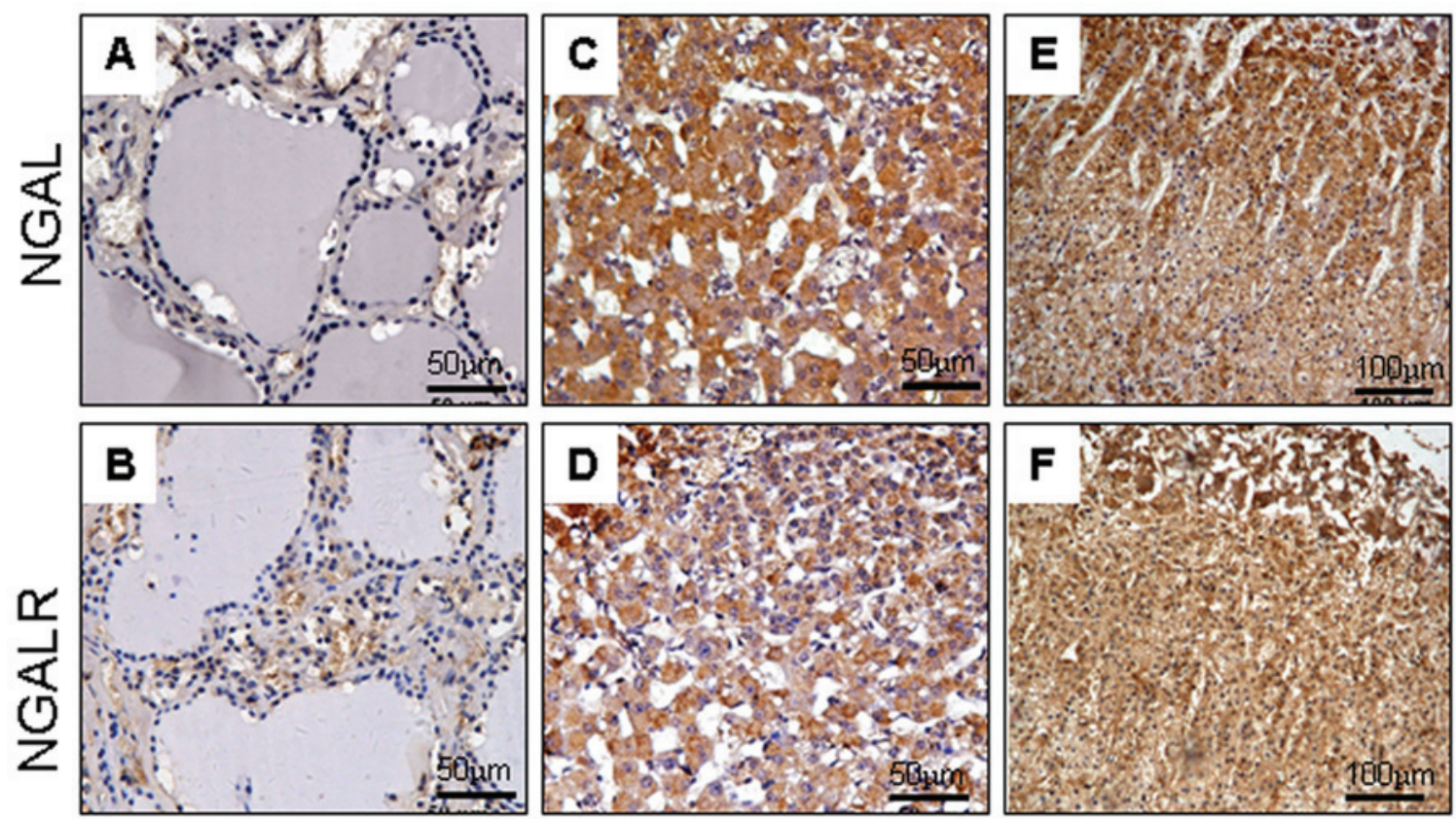

Figure 3. Expression of NGAL and NGALR in the endocrine system. (A and B) NGAL and NGALR reaection was not demonstrated in the follicular cells or C-cells of the thyroid gland. (C and D) In the adrenal gland, the cortex and medulla demonstrated a moderately positive reaction at 12 weeks' gestation. (E and F) In the later stages of development, NGAL was particularly present in cells of the inner and outer layers of the cortex and absent in the medulla, while NGALR exhibited strong positivity in the cortex and medulla. NGAL, neutrophil gelatinase-associated lipocalin; NGALR, neutrophil gelatinase-associated lipocalin receptor.
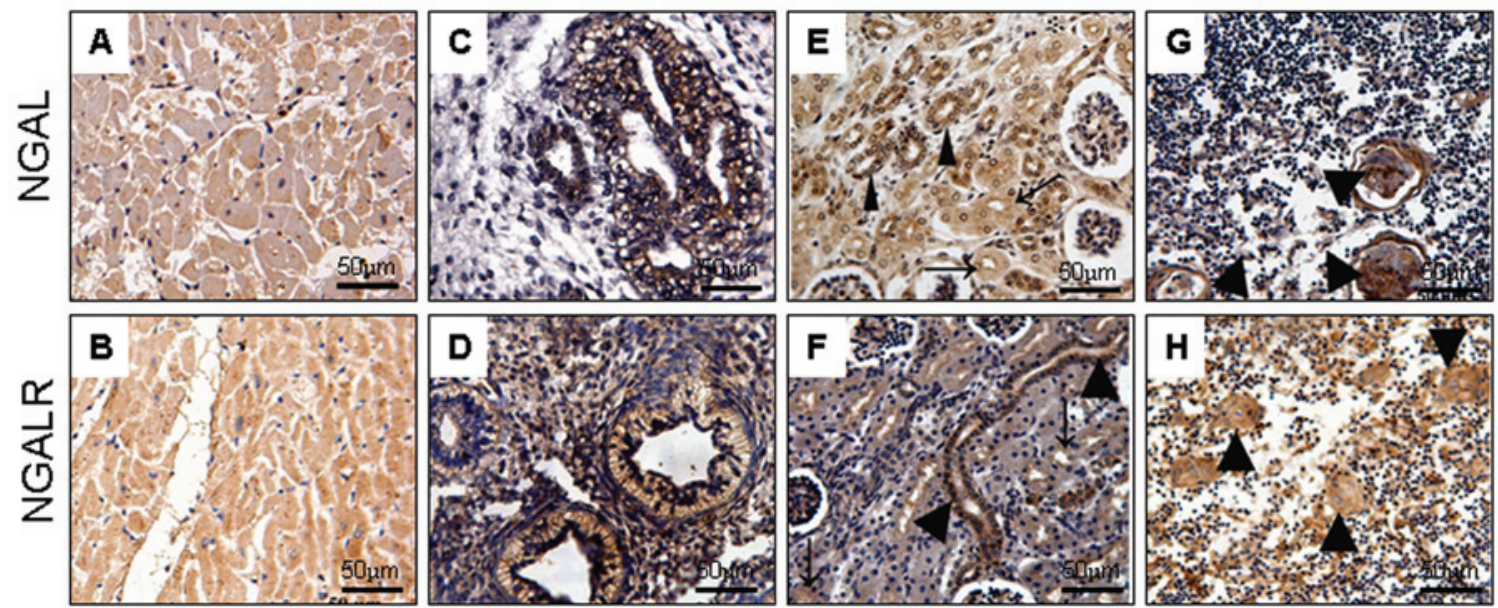

Figure 4. Expression of NGAL and NGALR in the other systems. (A and B) NGAL and NGALR demonstrated no or weak immunoreactivity in the heart tissue. (C and D) NGAL and NGALR were expressed in the epithelium of the alveoli gland at 12 weeks' gestation. (E and F) In the kidney, the proteins demonstrated weak positivity in the epithelial cells of the acinus renis, moderately positivity in the epithelium of the pars convoluta of the proximal tubule (arrows) and strong positivity in the the distal tubule (arrowheads) and collecting duct (arrowheads) at 22 weeks' gestation. (G and $\mathrm{H}$ ) In the thymus, the proteins were most prevalent in the subcapsular lymphocytes and the Hassall's corpuscle (arrowheads) epithelial cells. NGAL, neutrophil gelatinase-associated lipocalin; NGALR, neutrophil gelatinase-associated lipocalin receptor.

in the cells of the lung during later developmental stages. In the development of the genitourinary system, there was no evident difference between NGAL and NGALR expression. In the kidney, the two proteins were expressed in epithelial cells of the pars convoluta of the proximal tubule, and strongly expressed in epithelium of the distal tubule and the collecting duct (Fig. 4E and F). Bladder epithelium and prostate tissues were all negative. In the immune system, NGAL and NGALR were undetectable in the lymphocytes, but evidently demon- strated in the Hassall's corpuscle epithelial cells of the thymus (Fig. 4G and H).

\section{Discussion}

In the present study, we demonstrated that NGAL and NGALR were expressed in the epithelium of the renal tubule, the kidney, the thymus and the adrenal gland, but were not detectable in the heart, liver and thyroid gland, which was consistent with find- 
ings from a previous study demonstrating NGAL expression in normal human tissues (3). It was reported that in developing muscle fibers, a NGAL homologue was found in the hypertrophic cartilage during chicken embryo development (13) and in the hypertrophic region of growth plate cartilage in developing rat embryos (14). Mallbris et al investigated the expression of NGAL in human skin embryonic development and reported that the embryonic expression of NGAL was induced in the interfollicular epidermis at 20-24 weeks of gestational age, but thereafter progressively receded towards the hair follicles (21). In this study, we revealed that NGAL was expressed in the gastrointestinal tract during human embryo development. These findings suggest that NGAL is an evolutionarily conserved protein.

We found that NGAL and NGALR demonstrated different expression patterns in human embryonic, fetal and normal adult tissues, and their expression was adapted to become tissue and cell-specific throughout human development. The two proteins were weakly-positive or negative in normal cells of the thyroid gland, liver, heart and blood vessels, but they were abundant in the spleen and adrenal gland, and at the cell level, they were specific to lymphocytes and the epithelium of the renal tubule. In addition to a broad range of simple epithelial cell types, we found that the proteins were also expressed in stratified epithelia. For example, in the skin, mature cells of the outermost stratified layers exhibited a distribution of NGAL, which was inconsistent with results from a previous study (11). The expression of NGAL and NGALR does not appear to follow any morphogenetic pattern, however, colocalization with regards to cell function can be identified at closer examination.

Expression of NGAL and NGALR were also found to be time-specific during human growth and development. The proteins were expressed in the epithelium of the lung alveolus and gastrointestinal tract in the embryos, but were almost undetectable in the later developmental stages. In the embryonic adrenal gland, the cortex and medulla demonstrated a moderately positive reaction. In the adult tissues, NGAL was particularly present in the cells of the inner and outer layers of the cortex, but was absent in the medulla. Additionally, a pronounced expression pattern for NGAL was undetectable throughout the neural tube, but was detected in the stellate cells of the cerebrum and the Purkinje cells of the cerebellum in postnatal stages. It has been reported that the expression of NGAL was induced in the interfollicular epidermis at 20-24 weeks of gestational age, but thereafter progressively receded towards the hair follicles (21). Although there is no precise mechanism to explain the reason for the upregulation of the NGAL protein level in a number of normal tissues, as observed at early developmental stages, we speculate that NGAL might be involved in cell-cell interactions, cell division and/or cell growth, due to the high demand for those biological behaviors during development.

Lipocalins have been extensively used as biochemical markers for diseases. The clinical indications are associated with almost any field of medicine, including inflammatory disease, cancer, lipid disorders and kidney disease (22). Previous studies have demonstrated that overexpression of NGAL is involved in the progression of tumors (2,7-12). In cancers of the alimentary system, NGAL was upregulated in human colorectal and gastric cancer $(2,12)$. Results from our study revealed that the glandular epithelium of the normal adult gastrointestinal tract is negative for NGAL expression, but a pronounced positive staining was observed throughout the gastrointestinal tract at early fetal stages. Furthermore, NGAL was detected in the epithelium of the lung alveolus in embryos and A549 cells, a lung carcinoma cell line (6), but was almost undetectable in the adult lung tissue. Thus, NGAL immunoreactivity was found in cells of early fetal stages and in malignant cells, but was negative in the corresponding adult tissues, suggesting that there was a specific correlation between NGAL expression in certain malignant cells and in the corresponding normal cells from early developmental stages. We speculate that there may be a correlation between NGAL expression and the processes of embryogenesis and carcinogenesis.

In conclusion, the immunohistochemical observations in the present study are the first systematic demonstration of the immunoreactive presence of NGAL and NGALR in human embryonic, fetal and normal adult tissues. Through analyses of the differential expression, it was found that the expression of NGAL and NGALR is highly tissue- and time-specific.

\section{Acknowledgements}

The authors are grateful to Professor Xiao-Jun Yu from the Department of Forensic Medicine, Shantou University Medical College for his assistance in providing autopsy specimens. This study was supported by grants from the National High Technology Research and Development Program of China (No. 2006AA02A403), the Natural Science Foundation of China-Guangdong Joint Fund (No. U0932001) and the Foundation for Distinguished Young Talents in Higher Education of Guangdong, China (LYM09080).

\section{References}

1. Kjeldsen L, Johnsen AH, Sengeløv H and Borregaard N: Isolation and primary structure of NGAL, a novel protein associated with human neutrophil gelatinase. J Biol Chem 268: 10425-10432, 1993.

2. Nielsen BS, Borregaard N, Bundgaard JR, Timshel S, Sehested M and Kjeldsen L: Induction of NGAL synthesis in epithelial cells of human colorectal neoplasia and inflammatory bowel diseases. Gut 38: 414-420, 1996.

3. Friedl A, Stoesz SP, Buckley P and Gould MN: Neutrophil gelatinase-associated lipocalin in normal and neoplastic human tissues. Cell type-specific pattern of expression. Histochem J 31: 433-441, 1999.

4. Gwira JA, Wei F, Ishibe S, Ueland JM, Barasch J and Cantley LG: Expression of neutrophil gelatinase-associated lipocalin regulates epithelial morphogenesis in vitro. J Biol Chem 280: 7875-7882, 2005

5. Mori K, Lee HT, Rapoport D, Drexler IR, Foster K, Yang J, Schmidt-Ott KM, Chen X, Li JY, Weiss S, et al: Endocytic delivery of lipocalin-siderophore-iron complex rescues the kidney from ischemia-reperfusion injury. J Clin Invest 115: 610-621, 2005.

6. Tong Z, Wu X, Ovcharenko D, Zhu J, Chen CS and Kehrer JP: Neutrophil gelatinase-associated lipocalin as a survival factor. Biochem J 391: 441-448, 2005.

7. Bauer M, Eickhoff JC, Gould MN, Mundhenke C, Maass N and Friedl A: Neutrophil gelatinase-associated lipocalin (NGAL) is a predictor of poor prognosis in human primary breast cancer. Breast Cancer Res Treat 108: 389-397, 2008.

8. Moniaux N, Chakraborty S, Yalniz M, Gonzalez J, Shostrom VK, Standop J, Lele SM, Ouellette M, Pour PM, Sasson AR, et al: Early diagnosis of pancreatic cancer: neutrophil gelatinase-associated lipocalin as a marker of pancreatic intraepithelial neoplasia. Br J Cancer 98: 1540-1547, 2008. 
9. Monier F, Surla A, Guillot M and Morel F: Gelatinase isoforms in urine from bladder cancer patients. Clin Chim Acta 299 $11-23,2000$

10. Lim R, Ahmed N, Borregaard N, Riley C, Wafai R, Thompson EW, Quinn MA and Rice GE: Neutrophil gelatinase-associated lipocalin (NGAL) an early-screening biomarker for ovarian cancer: NGAL is associated with epidermal growth factor-induced epithelio-mesenchymal transition. Int J Cancer 120: 2426-2434, 2007.

11. Zhang H, Xu L, Xiao D, Xie J, Zeng H, Wang Z, Zhang X, Niu Y, Shen Z, Shen J, et al: Upregulation of neutrophil gelatinase-associated lipocalin in oesophageal squamous cell carcinoma: significant correlation with cell differentiation and tumour invasion. J Clin Pathol 60: 555-561, 2007.

12. Du ZP, Yuan HM, Wu BL, Chang JX, Lv Z, Shen J, Wu JY, Chen HB, Li EM and Xu LY: Neutrophil gelatinase-associated lipocalin in gastric carcinoma cells and its induction by TPA are controlled by C/EBP $\beta$. Biochem Cell Biol 89: 314-324, 2011.

13. Descalzi CF, Dozin B, Zerega B, Cermelli S and Cancedda R: Ex-FABP: a fatty acid binding lipocalin developmentally regulated in chicken endochondral bone formation and myogenesis. Biochim Biophys Acta 1482: 127-135, 2000.

14. Zerega B, Cermelli S, Michelis B, Cancedda R and Cancedda FD: Expression of NRL/NGAL (neu-related lipocalin/neutrophil gelatinase-associated lipocalin) during mammalian embryonic development and in inflammation. Eur J Cell Biol 79: 165-172, 2000.

15. Devireddy LR, Teodoro JG, Richard FA and Green MR: Induction of apoptosis by a secreted lipocalin that is transcriptionally regulated by IL-3 deprivation. Science 293: 829-834, 2001.
16. Fang WK, Xu LY, Lu XF, Liao LD, Cai, WJ Shen ZY and Li EM: A novel alternative spliced variant of neutrophil gelatinase-associated lipocalin receptor in esophageal carcinoma cells. Biochem J 403: 297-303, 2007.

17. Devireddy LR, Gazin C, Zhu X and Green MR: A cell-surface receptor for lipocalin $24 \mathrm{p} 3$ selectively mediates apoptosis and iron uptake. Cell 123: 1293-1305, 2005.

18. Cui L, Xu LY, Shen ZY, Tao Q, Gao SY, Lv Z, Du ZP, Fang WK and Li EM: NGALR is overexpressed and regulated by hypomethylation in esophageal squamous cell carcinoma. Clin Cancer Res 14: 7674-7681, 2008.

19. Du ZP, Lv Z, Wu BL, Wu ZY, Shen JH, Wu JY, Xu XE, Huang Q, Shen J, Chen HB, Li EM and Xu LY: Neutrophil gelatinase-associated lipocalin and its receptor: independent prognostic factors of oesophageal squamous cell carcinoma. J Clin Pathol 64: 69-74, 2011

20. Liu MF, Jin T, Shen JH, Shen ZY, Zheng ZC, Zhang ZL, Xu LY, Li EM and Xu HX: NGAL and NGALR are frequently overexpressed in human gliomas and are associated with clinical prognosis. J Neurooncol 104: 119-127, 2011.

21. Mallbris L, O'Brien KP, Hulthén A, Sandstedt B, Cowland JB, Borregaard N and Ståhle-Bäckdahl M: Neutrophil gelatinaseassociated lipocalin is a marker for dysregulated keratinocyte differentiation in human skin. Exp Dermatol 11: 584-591, 2002.

22. Xu S and Venge P: Lipocalins as biochemical markers of disease Biochim Biophys Acta 1482: 298-307, 2000.

23. Xie JJ, Zhang FR, Tao LH, et al: Expression of ezrin in human embryonic, fetal, and normal adult tissues. J Histochem Cytochem 59: 1001-1008, 2011. 